Originalien

Somnologie $2021 \cdot 25: 20-28$

https://doi.org/10.1007/s11818-020-00287-0

Angenommen: 3. November 2020

Online publiziert: 1. Dezember 2020

(c) Der/die Autor(en) 2020
Martin Glos ${ }^{1}$ Sandra Zimmermann ${ }^{1}$. Thomas Penzel ${ }^{1}$ Katharina Lederer ${ }^{1,2}$. Ingo Fietze ${ }^{1}$

${ }^{1}$ Interdisciplinary Sleep Medicine Center, Charité - Universitätsmedizin Berlin, Berlin, Deutschland

${ }^{2}$ Advanced Sleep Research GmbH, Berlin, Deutschland

\title{
Einfluss von abendlicher Computerspielnutzung auf Parameter der Alertness und des Schlafs bei Jugendlichen
}

\section{Einleitung}

Die Beschäftigung mit elektronischen Medien macht einen immer größeren Anteil am Freizeitverhalten von Kindern und Jugendlichen aus $[1,2]$ Damit ist auch die regelmäßige Computernutzung (einschl. Notebooks, Tablets u.ä. Handheld-Geräte) bei schulpflichtigen Kindern in Deutschland weit verbreitet. Die Verwendungsgebiete sind dabei umfangreich: von der Nutzung für schulische Belange, als allgemeine Informationsquelle, der Nutzung von sozialen Netzwerken bis hin zu Computerspielen unterschiedlichster Inhalte. Die gesundheitlichen Folgen der intensiven Mediennutzung sind umstritten. Es werden häufig psychosomatische Beschwerden und Verhaltensauffälligkeiten beschrieben [3]. Gerade der exzessive Konsum von Computerspielen wird mit vielen negativen Auswirkungen in Verbindung gebracht, so z. B. Aufmerksamkeits- und Merkfähigkeitsdefizite, Hyperaktivität und aggressives Verhalten [4]. Zu der Frage, ob sich die regelmäßige Computernutzung, v.a. in den Abendstunden auch auf den darauffolgenden Nachtschlaf auswirkt, existieren jedoch erst recht wenige internationale Studien [5]. Eine belgische Befragungsstudie mit 2546 Jugendlichen konnte zeigen, dass die ausgeprägte PC-Nutzung mit einer späteren Zubettgehzeit, einer früheren

Alle Autoren haben das Manuskript gelesen und genehmigt.
Aufstehzeit an Wochentagen und einer erhöhten Müdigkeit assoziiert ist [6]. Gaina et al. [7] fanden in einer großen Befragung $(N=9718)$ zusätzlich einen geschlechtsspezifischen Unterschied bzgl. negativer Auswirkungen von Computerspielen bei japanischen Schulkindern.

Es gibt wenige experimentelle Untersuchungen bei computernutzenden Kindern, in denen neben Befragungen zusätzlich auch objektive Messungen der Schlafquantität und der Schlafqualität durchgeführt wurden [4, 8, 9]. Diese wenigen Studien konnten jedoch übereinstimmend zeigen, dass eine übermäßige Nutzung von Medien (hierunter fällt auch das Fernsehen) zu einer verminderten Schlafqualität führen kann. Allerdings wurden in allen drei Untersuchungen ausschließlich Jungen untersucht, sodass die Übertragbarkeit der Ergebnisse auf das weibliche Geschlecht bisher nicht möglich ist. Der Grund für die selektive Stichprobenauswahl ist in dem weiblichen Zyklus und seinem Einfluss auf den Schlaf zu sehen. In der vorliegenden Studie sollen daher erstmals nun auch die Mädchen mitbetrachtet werden. Untersucht wird die Frage, inwieweit sich die abendliche 2-stündige Computernutzung (Computerspiel) im Vergleich zu einer 2-stündigen entspannten Lesesituation auf die Stimmung, auf die Befindlichkeit und Alertness sowie auf den darauffolgenden Nachtschlaf von heranwachsenden Mädchen und Jungen auswirkt.

\section{Methoden}

\section{Probanden}

In diese Pilotstudie wurden gesunde, deutschsprachige Schüler einer Berliner Realschulklasse mit insgesamt 32 Schülern (davon 11 männlich) im Alter von 14-15 Jahren zur Teilnahme an der Studie eingeladen. Ausschlusskriterien waren eine behandlungsbedürftige Schlafstörung, regelmäßiger Drogenund Alkoholkonsum, die regelmäßige Einnahme von Medikamenten sowie akute oder chronische behandlungsbedürftige Erkrankungen.

In dieser Studie wurden im Gegensatz $\mathrm{zu}$ bisherigen Untersuchungen hauptsächlich Mädchen untersucht. Da der weibliche Zyklus sowie die Verwendung von Kontrazeptiva einen Einfluss auf den Schlaf haben können, wurden diese Faktoren in Form einer separaten Befragung der weiblichen Studienteilnehmerinnen miterhoben.

\section{Experimentelles Design}

Einmalig wurden die Fragebögen Pittsburgh Sleep Quality Index PSQI [10] zur Erfassung von Schlafgewohnheiten und Schlafstörungen, Insomnia Severity Index (ISI) zur Erfassung von insomnischen Beschwerden [11], Epworth Sleepiness Scale (ESS) zur Erfassung von Einschlafneigung am Tage [12] sowie die deutsche Version des MorningnessEveningness-Questionnaire

(D-MEQ) 


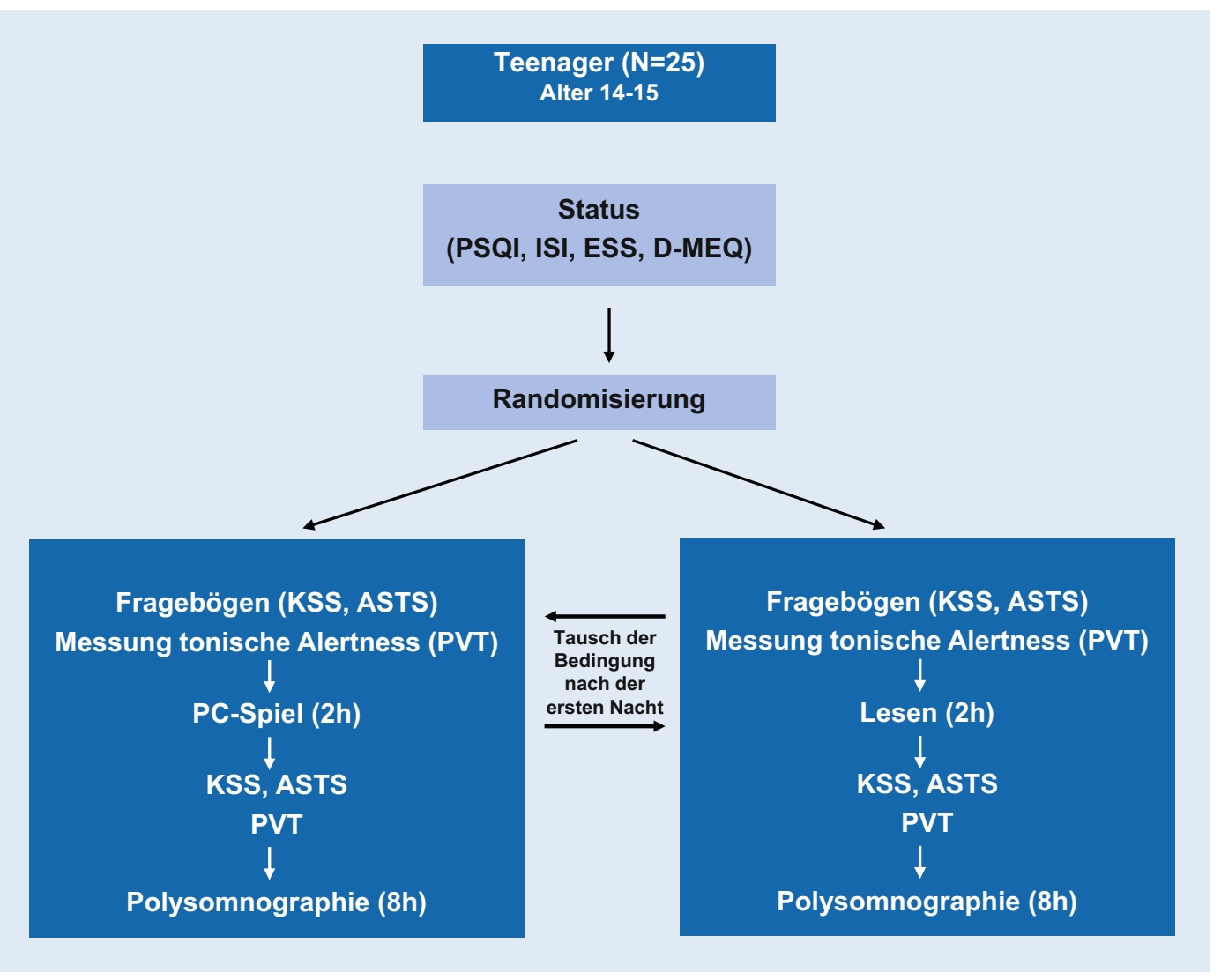

Abb. $1<$ Darstellung des Studienablaufs

zur Bestimmung des Chronotyps [13] erhoben.

Alle Jugendlichen wurden in einem DGSM-akkreditierten Schlaflabor für jeweils 2 aufeinanderfolgende Abende und anschließende Nächte mit Durchführung einer Polysomnografie (PSG) untersucht. Die zeitliche Abfolge der abendlichen Untersuchungen orientierte sich daran, eine einheitliche Licht-Aus-Zeit der PSG von 23:00 Uhr zu gewährleiste, welche sich am mittleren Wert der Selbstangabe der Jugendlichen orientiert. Es erfolgte eine Randomisierung der Probanden $\mathrm{zu}$ einer der beiden abendlichen Interventionen „Lesen“ oder „PC-Spiel“, welche für jeweils $2 \mathrm{~h}$ (19.00-21.00 Uhr) am ersten Abend durchgeführt wurden. Am zweiten Abend erfolgte dann die Durchführung der jeweils anderen Intervention im gleichen Zeitraum (• Abb. 1).

Vor Studienbeginn wurden für beide Interventionsbedingungen mit einem Luxmeter/mit Tubus (Mini-Lux, MX-Elektronik, Berlin) die wirksame Beleuchtungsstärke $\left(\mathrm{E}_{\mathrm{v}}\right)$ und die Leuchtdichte $\left(\mathrm{L}_{\mathrm{v}}\right)$ sowie mit einem Doppelmonochromator-Spektralradiometer
(OL 754, Optronic, Orlando, FL, USA) die spektrale Lichtverteilung zur Bestimmung der Farbtemperatur (CCT) gemessen. Unter der Lesebedingung (Deckenbeleuchtung an, sitzend, Blickrichtung lesend, $45^{\circ}$ nach unten) betrug die $\mathrm{E}_{\mathrm{v}}=1720 \mathrm{Lx}$, die $\mathrm{L}_{\mathrm{v}}=78,0 \mathrm{~cd} / \mathrm{m}^{2}$ und die $\mathrm{CCT}=2874 \mathrm{~K}$. Unter der Bedingung „PC-Spiel“ (Deckenbeleuchtung an, sitzend, Monitorentfernung $40 \mathrm{~cm}$ ) betrug die $\mathrm{E}_{\mathrm{v}}=2541 \mathrm{Lx}$, die $\mathrm{L}_{\mathrm{v}}=48,5 \mathrm{~cd} / \mathrm{m}^{2}$ und die $\mathrm{CCT}=2711 \mathrm{~K}$.

Das PC-Spiel war ein sogenanntes „Jump 'n' Run“-Computerspiel, welches die Jugendlichen für die Dauer der $2 \mathrm{~h}$ bei normaler Zimmerbeleuchtung ohne größere Unterbrechungen (z. B. in Form von längeren Pausen) spielen sollten.

Bei diesem Spiel handelt es sich um ein comicartiges Computerspiel, bei dem sich die Spielfigur laufend und springend fortbewegt und das präzise Springen einen wesentlichen Teil der spielerischen Handlung darstellt. Das Spiel („Ratatouille“, The Walt Disney Company, Burbank, CA, USA) ist vollkommen gewaltfrei.
Für die Lesebedingung erhielten die Teenager kommerzielle Jugendzeitschriften, mit denen sie sich separat in den Zimmern, bei identischer Beleuchtung und gleicher Zeitdauer beschäftigen konnten. Die Teenager durften während ihres Aufenthalts nicht fernsehen oder sich mit anderen Medien (z. B. dem Mobiltelefon/ Smartphone) beschäftigen.

Vor und nach den abendlichen Interventionen „Lesen“ oder „PC-Spiel“ wurden zusätzlich der Befindlichkeitsfragebogen Aktuelle Stimmungsskala (ASTS) [14] und der Fragebogen zur akuten Schläfrigkeit Karolinska Sleepiness Scale (KSS) [15] erhoben. Zur Messung der tonischen Alertness wurde der Psychomotor Vigilance Task (PVT) (PVT-192, Ambulatory Monitoring, Inc., Ardsley, NY, USA) ebenfalls zu beiden Zeitpunkten für jeweils 10 min durchgeführt und die mittlere Reaktionszeit (RT) und die Fehleranzahl (Err_num) ausgewertet.

Im Anschluss an die abendlichen Interventionen und Fragebogenerhebungen wurden die Jugendlichen für die PSG vorbereitet. Die Verkabelung erfolgte nach den AASM-Richtlinien [16]. 


\section{Glos · S. Zimmermann · T. Penzel · K. Lederer $\cdot$ I. Fietze}

\section{Einfluss von abendlicher Computerspielnutzung auf Parameter der Alertness und des Schlafs bei Jugendlichen}

\section{Zusammenfassung}

Hintergrund. Computerspielnutzung vor dem Schlafengehen ist vor allem bei Heranwachsenden ein übliches Verhalten. Die exzessive Nutzung kann mit somatischen Beschwerden, Aufmerksamkeitsdefiziten und familiären Interaktionsproblemen verbunden sein. Es gibt aber nur wenige Erkenntnisse über die Auswirkungen auf den nachfolgenden Schlaf. Fragestellung. Ziel dieser Pilotstudie war es, die Auswirkungen eines Computerspiels am Abend auf die Alertness und den Schlaf von Jugendlichen zu untersuchen.

Material und Methoden. Fünfundzwanzig gesunde Jugendliche (mittleres Alter 15 Jahre, $w=20, m=5$ ) aus einer Schulklasse wurden in diese Pilotstudie eingeschlossen. In einem randomisierten Crossover-Design an zwei aufeinanderfolgenden Tagen wurde jeweils am Abend für $2 \mathrm{~h}$ entweder ein Jump-andRun-Computerspiel durchgeführt oder eine Jugendzeitschrift gelesen. Vor und nach der jeweiligen Intervention wurde mittels Fragebögen die Stimmung (Aktuelle
Stimmungsskala, ASTS) und die Schläfrigkeit (Karolinska Sleepiness Scale, KSS) erhoben sowie Parameter der tonischen Alertness mittels Psychomotor Vigilance Task (PVT) gemessen. Jeweils im Anschluss wurde der Schlaf mittels Polysomnografie (PSG) untersucht.

Ergebnisse. Zweistündiges Computerspielen am Abend führte unmittelbar danach zu einer verringerten Alertness - der PVTParameter Reaktionszeit (RT) erhöhte sich von $272,0 \pm 30,5 \mathrm{~ms}$ auf $305,2 \pm 41,3 \mathrm{~ms}(p<0,01)$ während die RT im gleichen Zeitfenster am anderen Abend unter der Lesebedingung unverändert blieb.

Abendliche Computerspielnutzung führte in der darauffolgenden Nacht zu einem erhöhten Leichtschlafanteil (N1 + N2: 48,9 9, 9\%-TST vs. $44,6 \pm 9,8 \%$-TST, $p<0,05)$ und einem reduzierten Tiefschlafanteil (N3: 36,0 \pm 10,0\%TST vs. $39,5 \pm 9,0 \%$-TST, $p<0,05)$ gegenüber dem Schlaf nach zweistündigem Lesen. Die Einschlaflatenz und die Schlafeffizienz unterschieden sich zwischen beiden Bedingungen nicht.

Diskussion. Bei den in dieser Pilotstudie untersuchten gesunden Jugendlichen wirkten sich abendliche Computerspiele negativ auf die Alertness und die Schlafarchitektur der darauffolgenden Nacht aus. Zusammen mit häufig nicht ausreichenden Schlafzeiten an Schultagen bei Jugendlichen könnten kumulativ diese Befunde entwicklungsphysiologische Relevanz haben. Untersuchungen an Jugendlichen in weiteren Kollektiven mit entsprechender Gruppengröße sind jedoch notwendig, um die Ergebnisse weiter zu verifizieren und ggf. schlafhygienische Verhaltensempfehlungen für diese Altersgruppen entsprechend anzupassen.

Schlüsselwörter Jugendliche P PC-Nutzung · Schlafhygiene . Aufmerksamkeit · Einschlaflatenz

\section{Influence of computer game use in the evening on parameters of alertness and sleep in adolescents}

\section{Abstract}

Background. Playing computer games before bedtime is a common behavior, especially among adolescents. Excessive computer game use can be associated with somatic complaints, attention deficits, and family interaction problems. However, there is little knowledge about the effects of computer games on subsequent sleep.

Objectives. The aim of this pilot study was to investigate the effects of playing a computer game in the evening on the alertness and sleep of adolescents.

Materials and methods. Twenty-five healthy adolescents (mean age 15 years, $f=20, m=5$ ) from one school class were included in this pilot study. In a randomized crossover design on two consecutive days, either a jump-andrun computer game or reading a youth magazine was performed for $2 \mathrm{~h}$ in the evening. Before and after each intervention, mood
(ASTS) and drowsiness (KSS) were assessed by questionnaires and parameters of tonic alertness were measured by the psychomotor vigilance task (PVT). Subsequently, sleep was measured by polysomnography (PSG). Results. Two hours of computer gaming in the evening resulted in reduced alertness levels immediately thereafter: the PVT parameter response time (RT) increased from $272.0 \pm 30.5 \mathrm{~ms}$ to $305.2 \pm 41.3 \mathrm{~ms}(p<0.01)$ while it remained unchanged under the reading condition the other evening. In comparison to reading, using computer games in the evening led to an increased amount of light sleep (N1 + N2: 48.9 \pm 9.1\%TST vs. $44.6 \pm 9.8 \%$-TST, $p<0.05)$ and a reduced amount of deep sleep (N3: $36.0 \pm 10.0 \%$ TST vs. $39.5 \pm 9.0 \%$-TST, $p<0.05$ ) the following night. Sleep latency and sleep efficiency did not differ between the two conditions.
Conclusions. In the group of healthy adolescents examined in this pilot study, using computer games in the evening had immediate negative consequences on alertness and on sleep architecture the subsequent night. Together with frequently insufficient sleeping time on school days in adolescents, these findings cumulatively could have relevance for physiological development. Subsequent trials in different groups of adolescents with a sufficient number of subjects are needed in order to further verify these findings and possibly, to adapt sleephygiene behavioral recommendations for these age groups.

Keywords

Adolescent · PC use · Sleep hygiene · Attention . Sleep onset latency 
Die Aufzeichnungszeit betrug $480 \mathrm{~min}$ bei einer Bettzeit von 23.00-07.00 Uhr. Die PSGs wurden mit dem System Embla N7000 (Embla Inc., Broomfield, CO, USA) durchgeführt. Am Morgen nach der PSG konnten die Studienteilnehmer nach Erfüllen der Studienbedingungen (Fragebogen ausfüllen und Aufmerksamkeitstest bearbeiten) die Einrichtung verlassen, um ihren gewohnten Aktivitäten nachzugehen.

Der Konsum von Kaffee oder anderen wachhaltenden Substanzen war den Jugendlichen für die gesamte Dauer der Studientage untersagt. Sowohl die Sorgeberechtigten der Schüler als auch die Schüler selbst mussten der Teilnahme schriftlich zustimmen. Die Studie wurde durch die Ethikkommission der CharitéUniversitätsmedizin Berlin genehmigt (EA 1/288/10).

\section{Statistik}

Alle Daten wurden mit der Software SPSS (IBM Corp., Amonk, NY, USA) statistisch ausgewertet. Mittelwertunterschiede wurden mit nichtparametrischen Tests (Wilcoxon; Mann-Whitney) für abhängige Stichproben auf Signifikanz getestet. Zur Untersuchung eines möglichen Carry-Over-Effekts wurde der Mann-Whitney-Test zwischen den Summenwerten der Sequenzgruppen „PC-Test“-,Lesen“ und „Lesen“-,PC-Test“ für die Schlafvariablen durchgeführt. Das Signifikanzniveau wurde auf $p \leq 0,05$ festgelegt.

\section{Ergebnisse}

\section{Probanden}

Von den eingeladenen Schülern der Klasse lehnten $N=7$ die Teilnahme entweder aus mangelndem Interesse oder aufgrund der fehlenden Einwilligung der Eltern ab. Es wurden $N=20$ Mädchen und $N=5$ Jungen im durchschnittlichen Alter von $14,7 \pm 0,4$ Jahren eingeschlossen. Bis auf eine Jugendliche waren alle Teilnehmer mit einem durchschnittlichen BMI von 20,0 $\pm 2,4$ normalgewichtig.

Die Teilnehmer gaben an, den Computer werktags durchschnittlich für $164,5 \pm 75,0$ min zu nutzen. Die Mädchen nutzen den PC dabei im Schnitt 40 min weniger als die Jungen: $154,7 \pm 72,7$ vs. $198,0 \pm 81,1$, dieser Unterschied erwies sich jedoch als nicht signifikant. Für das Wochenende gaben die Teilnehmer eine durchschnittliche Nutzungsdauer von $228,1 \pm 170,3 \mathrm{~min}$ an. Es gaben $59,1 \%$ $(N=13)$ der Jugendlichen an, den PC werktags wie auch am Wochenende am häufigsten in den Abendstunden zu nutzen, nachmittags $36,4 \%(N=8)$ und nachts $4,5 \%(N=1)$. Von einer Person wurde hierzu keine Angabe gemacht.

Die Heranwachsenden nutzen den Computer hauptsächlich für Aktivitäten im Internet $(95,2 \%, N=20)$; ein Studienteilnehmer verwendete diesen vorwiegend zur Spiele-Nutzung.

Anhand des D-MEQ wurde zusätzlich der Chronotyp der Teilnehmer ermittelt. Hier zeigte sich eine Normalverteilung der 5 möglichen Typen: 15 Neutraltypen, 3 moderate Morgen- sowie Abendtypen und 2 definitive Abendtypen. Definitive Morgentypen konnten nicht ermittelt werden.

Die separate Befragung der weiblichen Probanden erbrachte, dass $N=14$ Mädchen $(82,4 \%)$ zum Studienzeitpunkt bereits einen regelmäßigen Menstruationszyklus hatten. Von keiner der Teilnehmerinnen wurden zu diesem Zeitpunkt regelmäßig hormonelle Verhütungsmittel genutzt.

\section{Subjektive Schlaf- und Befindlichkeitsparameter}

Die Probanden gaben an, werktags gewöhnlich gegen 22:40 Uhr $( \pm 2: 20 \mathrm{~h}) \mathrm{zu}$ Bett zu gehen. Die mittlere Gesamtschlafdauer liegt bei 6,9 $\pm 1,2 \mathrm{~h}$. Am Wochenende verschiebt sich die Bettzeit der Teenager erwartungsgemäß nach hinten. Die mittlere Zubettgehzeit liegt hier in etwa bei 2.00 Uhr. Es zeigt sich eine negative signifikante Korrelation zwischen dem BMI und der von den Jugendlichen selbst angegebenen durchschnittlichen Gesamtschlafzeit (Total Sleep Time, TST) von $-0,43(p=0,05)$.

Fast alle Studienteilnehmer klagten über Einschlafschwierigkeiten gemessen anhand des ISI: leicht: $(N=9)$; mäßig: $27,3 \%(N=6)$, schwer: $18,2 \quad(N=4)$; sehr schwer: $9,1 \%(N=2)$; lediglich ein Jugendlicher berichtet über keinerlei
Hier steht eine Anzeige. 글 Springer 
Einschlafschwierigkeiten. Durchschlafschwierigkeiten wurden dagegen prozentual geringer angegeben: keine: 40,9\% $(N=9)$, leichte bis sehr schwere Durchschlafschwierigkeiten: 59,1\% $(N=13)$. Über eine Beeinträchtigung im Alltag durch die vorhandenen Schlafprobleme klagen allerdings nur $N=2$ Jugendliche und zeigen sich darüber zu einem großen Teil auch nicht besonders besorgt: keine Sorgen: $42,9 \%(N=9)$, wenig bis mäBige Sorgen: $47,6 \%(N=10)$, ziemliche Sorgen: $9,5 \%(N=2)$.

Die ESS betrug im Mittel 7,4 5,6. Bei $31,7 \%(n=7)$ der Jugendlichen war der ESS-Wert $\geq 10$. Die ESS zeigte dabei einen positiven Zusammenhang mit der täglichen Nutzungsdauer des PC $(r=0,43$, $p=0,05)$, der bei den Mädchen noch ausgeprägter war $(r=0,53 ; p=0,02)$.

Bezüglich des subjektiven Müdigkeitsgrades gab es keine relevanten Unterschiede innerhalb und zwischen den Gruppen sowie auch nicht geschlechtsspezifisch.

Es zeigten sich auch keine auffälligen Mittelwertdifferenzen hinsichtlich der aktuellen Stimmungslage, weder innerhalb der Gruppen (jeweils vor und nach einer Bedingung) noch beim Vergleich nach dem PC Spiel mit dem Lesen. Männliche Jugendliche tendierten nach dem Computerspiel zwar zu einem verstärkten Trauerempfinden in der ASTSGesamtskala (PC: vor: $4,20 \pm 1,8$ vs. nach: 7,0 $\pm 5,5$; Lesen: vor: $4,40 \pm 1,7$ vs. nach: 4,8 $\pm 2,2$ ), diese Tendenz erwies sich jedoch als nicht signifikant.

\section{Schlafstruktur}

Es konnten bei $n=23$ Probanden jeweils beide PSGs ausgewertet werden, die Datensätze von zwei Probanden mussten aufgrund von technischen Problemen bei der PSG-Aufzeichnung von der Analyse ausgeschlossen werden.

Es wurden keine Carry-Over-Effekte zwischen den beiden Sequenzgruppen „PC-Test-Lesen“ und „Lesen - PC-Test“ gefunden.

Insgesamt wurde bei allen Teilnehmern folgende mittlere Gesamtschlafzeiten (TST) gemessen: die Schlafzeit nach dem Lesen lag bei $M=409,9 \pm 55,5 \mathrm{~min}$, nach dem PC-Spiel bei 411,1 $\pm 36,1 \mathrm{~min}$. (s. - Tab. 1). Die Einschlaflatenz zu N2 (Sleep Onset Latency, SOL) nach dem Lesen zeigte sich gering höher als die Schlaflatenz nach dem PC-Spielen: $33,3 \pm 22,1$ vs. $29,5 \pm 20,5 \mathrm{~min}$.

Die Wachzeiten (WASO, Min.) waren nach dem PC-Spiel gegenüber der Lesevariante geringfügig erhöht, jedoch statistisch nicht signifikant. Beim Vergleich der Mittelwerte der quantitativen Schlafvariablen fanden sich keine geschlechtsspezifischen Unterschiede.

Der Leichtschlafmenge war nach dem PC-Spiel signifikant erhöht (• Abb. 2).

Dieselbe Tendenz zeigte sich auch bei alleiniger Betrachtung der Mädchen $(183,1 \pm 40,7$ vs. $196,0 \pm 43,8 \mathrm{~min})$, allerdings statistisch nicht signifikant. Auch der prozentuale Anteil an Leichtschlaf der TST unterschied sich zwischen den beiden Bedingungen bedeutsam, bzw. für die Mädchen auch hier wiederum nur ein Trend hinsichtlich zunehmender Mittelwerte nach dem PC-Spiel: $43,9 \pm 10,1$ vs. $47,3 \pm 9,3 \%$ TST $(p=0,07)$.

Der Tiefschlaf (N3) war in der Gesamtgruppe nach dem PC-Spiel gegenüber der Lesevariante signifikant reduziert, sowohl absolut als auch prozentual. Für die Mädchen allein ergab sich hier wiederum nur der Trend in diese Richtung (Lesen: 168,4 $\pm 43,4$ vs. PC: $153,3 \pm 43,0)$. Bei Betrachtung des REM-Schlafs (Min) innerhalb der Gesamtgruppe gab es lediglich eine geringe Tendenz einer reduzierten REM-SchlafZeit nach dem PC-Spiel; der prozentuale REM-Anteil (TST) blieb dagegen unverändert, ebenso die Schlafeffizienz (SE).

\section{Alertness}

Innerhalb der Lesebedingung gibt es keine statistisch relevanten Unterschiede in der RT beim Vergleich „vor/nach Lesen“: vor Lesen: $278,0 \pm 42,2 \mathrm{~ms}$ vs. nach Lesen: $276,8 \pm 45,5 \mathrm{~ms}$. In der PC-Variante zeigten sich dagegen signifikante Unterschiede in der RT beim Vergleich vor/ nach dem PC-Spiel: vor dem PC-Spiel lagen die RT bei $272,0 \pm 30,5 \mathrm{~ms}$, während sie nach PC-Spiel auf 305,2 $\pm 41,3 \mathrm{~ms}$ anstieg $(p=0,00)$.

Zusätzlich zur RT wurde auch die Err_num über den 10-minütigen Zeit- raum vor und nach den Bedingungen statistisch analysiert. Hier ergaben sich ähnliche Ergebnisse wie bereits bei der RT: Während sich die Err_num vor und nach der Lesebedingung nicht relevant unterschied (vor Lesen: 5,6 \pm 9,3 vs. nach Lesen: $6,7 \pm 7,1$ ), zeigte sich bei der PCSpiel Variante auch eine erhöhte Err_num nach dem Spiel (vor PC: $4,1 \pm 4,0$ vs. nach PC: $8,9 \pm 10,2 ; p=0,01)$.

\section{Diskussion}

In dieser Pilotstudie wurde untersucht, inwieweit sich die abendliche 2-stündige Computernutzung im Vergleich zu einer 2-stündigen entspannten Lesesituation auf die Stimmung, die Befindlichkeit, die Reaktionsschnelligkeit sowie auf den darauffolgenden Nachtschlaf von jugendlichen Schülerinnen und Schülern auswirkt.

Die insgesamt 23 Probanden wurden zudem zu ihren Gewohnheiten bzgl. der täglichen PC-Nutzung befragt. Die tägliche durchschnittliche Nutzungsdauer des PC von fast $3 \mathrm{~h}$ am Nachmittag/ Abend während der Woche zeigt, dass die Internetnutzung erwartungsgemäß eine bedeutsame Rolle im Alltag der Teenager spielt. Auch andere Studien konnten über einen ähnlich hohen Konsum dieser und anderer Medien, bezogen auf die tägliche Bildschirmzeit, berichten $[6,17]$. Dieses Ergebnis unterstreicht die Wichtigkeit, den Einfluss dieser Medien auch auf die Schlaf- und Befindlichkeitsparameter genauer zu untersuchen. Laut einer Fragebogenstudie des Robert Koch-Instituts gaben $76 \%$ von über 6813 befragten Kindern im Alter von 11-17 Jahren an, täglich einen Computer zu nutzen [18]. Dabei nutzten 34,4\% der befragten Jungen und Mädchen den Computer dabei mindestens 1-2h am Tag.

Einige Befragungen [17] konnten zudem zeigen, dass Mädchen den PC im Vergleich zu Jungen weniger häufig nutzen. Ein ähnlicher Trend wurde auch in der vorliegenden Untersuchung sichtbar: Die Mädchen nutzen den PC werktags durchschnittlich $40 \mathrm{~min}$ weniger als die Jungen. Allerdings bleibt dieses Ergebnis ohne statistische Relevanz. Die tägliche Nutzungsdauer des PC korrelier- 
Tab. 1 Mittelwerte (MW) und Standardabweichungen (SD) der Schlafparameter nach dem abendlichen 2-stündigen Lesen oder PC-Spiel $(N=23)$

\begin{tabular}{llll} 
Parameter & $\begin{array}{l}\text { Lesen } \\
\text { MW (SD) }\end{array}$ & $\begin{array}{l}\text { PC-Spiel } \\
\text { MW (SD) }\end{array}$ & p \\
\hline SOL (min) & $33,3(22,1)$ & $29,5(20,5)$ & 0,74 \\
\hline N1 + N2 (min) & $182,9(42,9)$ & $201,0(40,2)$ & $\mathbf{0 , 0 4}$ \\
\hline N1 + N2 (\%) & $44,6(9,8)$ & $48,9(9,1)$ & $\mathbf{0 , 0 1}$ \\
N3 (min) & $161,4(41,0)$ & $147,4(40,8)$ & $\mathbf{0 , 0 5}$ \\
N3 (\%) & $39,5(9,0)$ & $36,0(10,0)$ & $\mathbf{0 , 0 2}$ \\
REM (min) & $66,0(24,2)$ & $62,6(20,6)$ & 0,35 \\
REM (\%) & $15,9(5,1)$ & $15,1(4,5)$ & 0,25 \\
TST (min) & $409,9(55,5)$ & $411,1(36,1)$ & 0,58 \\
WASO (min) & $29,1(25,1)$ & $27,9(17,8)$ & 0,55 \\
SE (\%) & $86,2(9,7)$ & $87,4(6,3)$ & 0,78 \\
\hline $\begin{array}{l}\text { p Signifikanz, SOL Sleep Onset Latency, REM Rapid Eye Movement, TST Total Sleep Time, } \\
\text { WASO Wake after Sleep Onset, SE Schlafeffizienz }\end{array}$ \\
\hline
\end{tabular}

te in unserer Untersuchung wie auch in einer japanischen und belgischen Querschnittsstudie $[6,7]$ mit der Ausprägung der Tagesschläfrigkeit: Je länger die tägliche Nutzungsdauer des PC, desto stärker erwies sich auch die berichtete Schläfrigkeit. Bei alleiniger Betrachtung der Mädchen wurde dieses Ergebnis sogar noch eindrücklicher. Gaina et al. konnten diesen positiven Zusammenhang dagegen lediglich für das männliche $\mathrm{Ge}$ schlecht nachweisen [7]. Bereits ein Drittel der Jugendlichen der vorliegenden Untersuchung berichtet über eine auffällige Tagesmüdigkeit. Verstärkte Schläfrigkeit und deren negative Auswirkungen auf die täglichen Aktivitäten sowie die Leistungsfähigkeit konnten auch Wolfson et al. [19] und Gaina et al. [7] nachweisen. Eine durchschnittliche Schlafzeit von $<6,5 \mathrm{~h}$ stand erwartungsgemäß mit einer erhöhten Schläfrigkeit im Zusammenhang. Einige Autoren nehmen an, die Ursache für die hohen Schläfrigkeitswerte liege vor allem in der nach hinten verschobenen Zubettgehzeit durch die längere Nutzungsdauer der Computer in den Abendstunden $[6,20]$. In unserer Studie konnte gezeigt werden, dass die Jugendlichen auch während der Woche sehr spät ins Bett gehen.

Andere wiederum sehen die eigentliche Ursache nicht in den abendlichen Aktivitäten, sondern in der pubertär bedingten verzögerten Ausschüttung von Melatonin am Abend, der daraus resultierenden verschobenen Bettzeit und dem durch den festgelegten Schulbeginn bedingten frühen Aufstehen [21, 22]. Die Verteilung der Chronotypen zeigte keine auffälligen Ausprägungen. Die Mehrzahl der Teilnehmer befanden sich im „Neutraltyp“-Bereich, sodass eine Verzögerung der abendlichen Bettzeiten nicht zusätzlich mit dem Vorliegen vermehrter Abendtypen zurückzuführen ist. Eine solche Verteilung wurde aufgrund der in diesem Alter vorherrschenden Tendenz zum Morgentyp [23, 24] jedoch auch nicht erwartet. Letztendlich muss auf die unterschiedlichen Erhebungsmethoden und Definitionen verschiedener Studien von Müdigkeit/Schläfrigkeit hingewiesen werden, die einen direkten Vergleich erschweren.

\section{Subjektive Schlaf- und Befindlichkeitsparameter}

Die gewöhnlichen Schlafzeiten der Teilnehmer scheinen insgesamt verkürzt. Die jugendlichen Schüler schlafen mit 6,9h während der Woche zu wenig, was sich auch in den erhöhten Werten der Tagesschläfrigkeit basierend auf der ESS widerspiegelt. Gaina et al. [7] fanden bzgl. der Vermeidung von Tagesschläfrigkeit eine optimale Schlafdauer von 7,5-8h. Schlafzeiten unter $6,5 \mathrm{~h}$ gingen dagegen mit einer starken Schläfrigkeit einher. Tarokh et al. [25] geben in einem Review zur Schlafphysiologie bei Heranwachsenden, welcher sich auch auf eine Studie von Ohayon et al. [26] bezieht, eine mittle- re Schlafdauer von etwa 9,25h an, welche über einen langen Altersbereich von 10-12 bis 15-18 Jahren relativ konstant bleibt. Diese Werte wurden allerdings nur gemessen, wenn eine Bettzeit von $10 \mathrm{~h}$ zur Verfügung steht. Andererseits stimmen die subjektiven Angaben bzgl. der durchschnittlichen Gesamtschlafzeit auch mit der objektiv gemessenen TST im Schlaflabor während der Studie überein. Am Wochenende verschiebt sich die Bettzeit erwartungsgemäß noch zusätzlich nach hinten. Auch diese Ergebnisse können mit der Annahme der verspäteten Melatoninausschüttung und dadurch verzögerter Zubettgehzeit bei jugendlichen Heranwachsenden erklärt werden.

Ein weiteres interessantes Ergebnis ist, dass die Studienteilnehmer bereits in der Überzahl über verstärkte Einschlafschwierigkeiten berichten, sich dadurch jedoch scheinbar nicht weiter beeinträchtigt fühlen oder sich darüber Sorgen machen, obwohl ein Drittel bereits über eine besorgniserregende Schläfrigkeit berichtet. Bei Vergleich der aktuellen Schläfrigkeit (KSS) vor und nach den experimentellen Bedingungen ließen sich jedoch keine relevanten Veränderungen feststellen, auch nicht bei getrennter Betrachtung der Geschlechter.

Li et al. [27] berichten bei chinesischen Schulkindern über eine gesteigerte Angst und Unruhe vor dem Schlafengehen, wenn ein erhöhter abendlicher PCund Fernsehkonsum gegeben ist. In unserer Studie erbrachte die Messung der aktuellen Stimmung dagegen keine Auffälligkeiten, außer der leichten Tendenz der männlichen Jugendlichen nach dem PCSpielen eine verstärkte Trauer in der Gesamtskala zu empfinden, was sich jedoch als nicht signifikant erwies. Letztendlich werden die Stimmungslagen nach PCSpielen jedoch stark von ihrem Inhalt abhängen. In unserer Studie wurde lediglich eine gewaltfreie Variante angeboten, deren affektiver Effekt weniger bedeutend sein dürfte. Die Ergebnisse der chinesischen Studie beziehen sich zudem auf eine jüngere Altersgruppe.

\section{Schlafstruktur}

Insgesamt wurde bei den Teilnehmern nach beiden experimentellen Bedingun- 


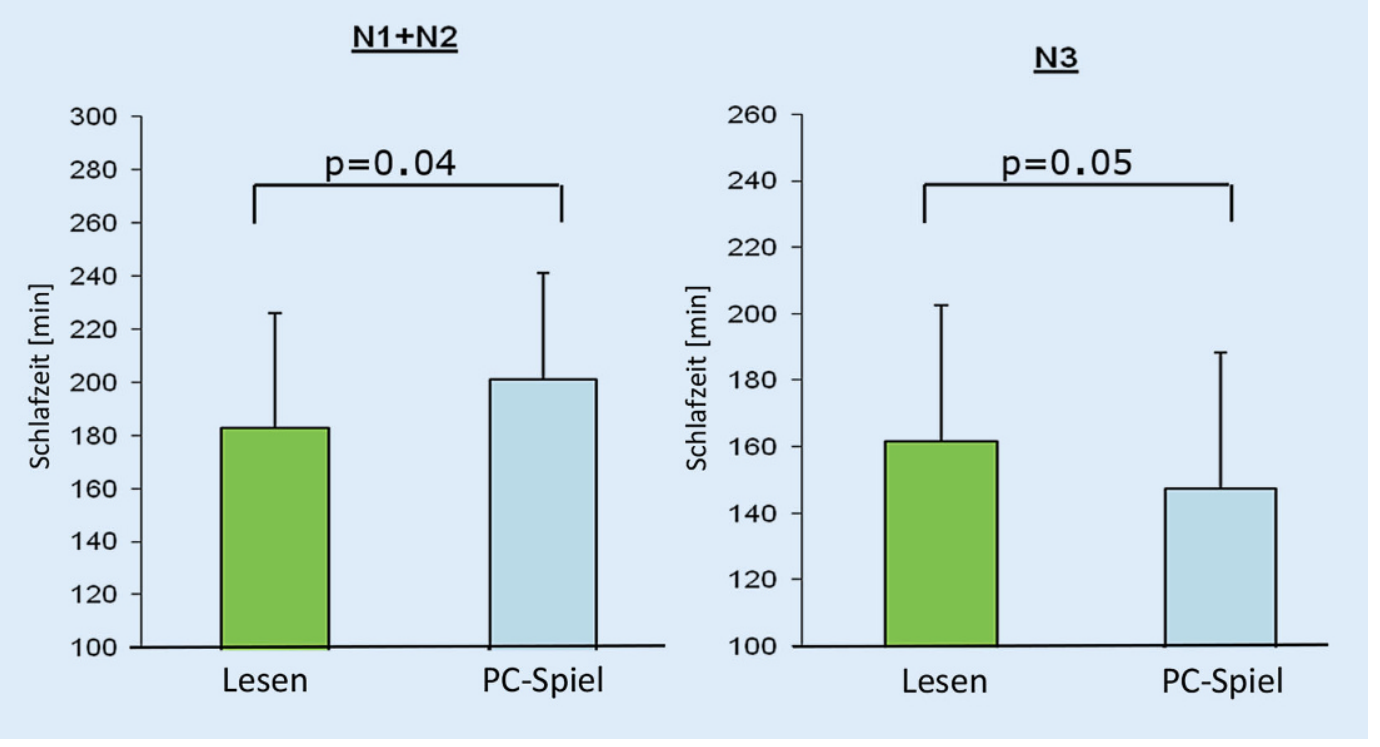

Abb. $2<$ Vergleich von Leicht- (N1 + N2)- und Tiefschlafmenge (N3) nach dem abendlichen, jeweils 2-stündigen Lesen oder PCSpiel gen eine ähnliche Gesamtschlafzeit gemessen. Die Schlafzeit zeigte sich trotz der eingehaltenen Bettzeit von $8 \mathrm{~h}$ mit $6,9 \mathrm{~h}$ insgesamt reduziert.

Die Schlaflatenz zum Schlafstadium N2 (SOL) war unter beiden Bedingungen erhöht, jedoch nicht wie in anderen Studien berichtet, nur nach dem Medienkonsum $[4,8]$. Auch in der Studie von Weaver et al. [9] konnten nur sehr geringe Unterschiede in der SOL gefunden werden. Allerdings handelt es sich hier um kaum vergleichbare Ergebnisse, da die Jugendlichen neben der unterschiedlichen Nationalität ein durchschnittlich höheres Alter von 16,6 Jahren aufwiesen und ausschließlich männlichen Geschlechts waren. Eine abendliche Beleuchtungsstärke ab 2500 Lx soll einigen Studien zufolge die Einschlaflatenzen verlängern [28, 29]. Dabei wird, bedingt durch den geringeren Abstand zum Auge bei Computermonitoren im Vergleich zum klassischen abendlichen Fernsehkonsum, ein größerer Einfluss auf die objektiven Schlafparameter vermutet [4].

In unserer Studie betrug die gemessene Beleuchtungsstärke unter der Bedingung PC-Spiel $2541 \mathrm{Lx}$ im Vergleich zu $1720 \mathrm{Lx}$ unter der Lesebedingung. Höhere Einschlaflatenzen traten beim PCSpiel jedoch nicht auf; möglicherweise, weil die Beleuchtungsstärke in beiden $\mathrm{Be}$ dingungen recht hoch war. Ähnliche Befunde berichtet Higuchi et al. [8] welche nur minimale Unterschiede in der SOL bei verschieden hellen Displays fanden. Die insgesamt verzögerte Einschlaflatenz könnte zudem auch durch die ungewohnten Laborbedingungen bedingt sein.

Die Anzahl der Weckreaktionen zeigte sich nach dem PC-Spiel zwar leicht erhöht, ist jedoch statistisch nicht bedeutsam, was auch anderen Studienergebnissen entspricht [4]. Auch bezüglich der Schlafeffizienz findet sich innerhalb und zwischen den Gruppen kein Unterschied. Wie schon bei Dworak et al. fand sich bei der Gesamtgruppe nach dem PCSpiel jedoch ein signifikant reduzierter Tiefschlaf, sowohl in Minuten als auch anteilig am Gesamtschlaf gemessen. Der Leichtschlafanteil war demgegenüber bedeutsam erhöht. Für den REM-Schlaf konnte lediglich eine geringe Tendenz der Verkürzung nach dem PC-Spiel beobachtet werden. Auch diese Ergebnisse stimmen mit Dworak et al. [4] überein.

Die vorliegende Reduktion von Tiefschlaf zugunsten des Leichtschlafs nach der Intervention „PC-Spiel“ ist aufgrund nicht erhöhter Einschlaflatenzen vermutlich keine Folge einer lichtbedingten Verzögerung in der Melatoninausschüttung. Ob die kognitive Beanspruchung durch das PC-Spiel einen singulären Einfluss auf den Tiefschlaf hat, kann aus den vorliegenden Daten nicht beantwortet werden und müsste in weiteren Studien untersucht werden.

\section{Alertness}

Die Ergebnisse des PVT lassen vermuten, dass ein abendliches Computerspiel bei Jugendlichen ausreicht, die objektive Aufmerksamkeit zu reduzieren. Dafür spricht das Absinken der mittleren 10-minütigen Reaktionszeit $R T$ nach der PC-Variante. Würde eine erhöhte kognitive Alertness durch zuvor stark aufmerksamkeitsfordernde Bedingungen vorliegen, würde die RT verkürzt sein, wie es bei anderen Studien gefunden werden konnte [30]. Jedoch untersuchten diese meist keine unmittelbaren Effekte wie in unserer Studie, sondern verglichen regelmäßig computerspielende Personen mit Nichtspielenden. Innerhalb unserer Studie haben die spezifischen inhaltlichen Bedingungen des ausgewählten Spiels bei den Jugendlichen scheinbar eher zu einer „Ermüdung“ als zu der erwarteten erhöhten Alertness geführt. Im Gegensatz zu anderen Untersuchungen [4] wurde das Computerspiel in unserer Studie zudem am Abend als auch über einen längeren Zeitraum ( $2 \mathrm{~h}$ ) durchgeführt, was die verringerte Alertness ebenfalls erklären könnte.

\section{Limitationen der Studie}

Die Studie wurde als Pilotstudie mit einer entsprechend limitierten Fallzahl konzipiert und ist daher in ihrer statistischen Aussagekraft begrenz. Größere prospek- 
tive Fallkontrollstudien mit entsprechender Fallzahlplanung und Alpha-Adjustierung sind daher notwendig, um die in dieser Studie gefundenen Hinweise - u. a. mit Angabe von Effektstärken weiter verifizieren zu können. Es konnte in dieser Studie aus schulorganisatorischen Gründen nicht an drei aufeinanderfolgenden Tagen untersucht werden; somit wurde zusätzlich keine BaselinePSG durchgeführt. Obwohl kein CarryOver-Effekt nachgewiesen werden konnte, sollten zukünftige Untersuchungen eine Baselinemessung beinhalten. Die untersuchte Gruppe von Schülern war homogen; es wurden nur Schüler einer Klasse aus einer Schule untersucht. Zukünftige Studien sollten heterogene Gruppen einschließen um den Einfluss von Alter, Sozialstatus, Schultyp und anderen demografischen Faktoren berücksichtigen zu können. Diese Studie war eine Laborstudie mit kontrollierten Bedingungen, jedoch lassen sich die Ergebnisse nicht direkt auf Alltagssituationen übertragen. Daher sollte das Untersuchungsziel zukünftig auch durch Feldstudien ergänzt werden.

\section{Schlussfolgerungen}

Die Ergebnisse dieser Pilotstudie geben Hinweise darauf, dass abendliche Computerspiele einen unmittelbaren Einfluss auf die Alertness und den nachfolgenden Schlaf haben könnten. Vor dem Hintergrund einer bei Jugendlichen an Schultagen häufig verkürzten Schlafdauer könnten diese Ergebnisse kumulativ entwicklungsphysiologische Relevanz haben. Weitere Untersuchungen mit größeren Fallzahlen und in heterogenen Kohorten sind jedoch notwendig, um diese Erkenntnisse weiter zu verifizieren und ggf. bei der Gestaltung von Aufklärungs- und Präventionsprogrammen zum Thema "Gesunder Schlaf bei Jugendlichen“ zu berücksichtigen.

\section{Korrespondenzadresse}

\section{Dr. Martin Glos}

Interdisciplinary Sleep Medicine Center, Charité - Universitätsmedizin Berlin Charitéplatz 1, 10117 Berlin, Deutschland martin.glos@charite.de
Danksagung. Die Autoren danken Dr. rer. nat. Helmut Piazena, Charité-Universitätsmedizin Berlin, AG Photobiologie für die Durchführung und Auswertung der Lichtmessungen.

Funding. Open Access funding enabled and organized by Projekt DEAL.

\section{Einhaltung ethischer Richtlinien}

Interessenkonflikt. I. Fietze erhielt Forschungszuschüsse von Actelion, Eisai, Heinen \& Löwenstein Jazz Pharmaceuticals, Philips/Respironics, Resmed, Somnodent, UCB, Vanda. M. Glos erhielt einen Forschungszuschuss von Phasya. T. Penzel erhielt Forschungszuschüsse von Heinen \& Löwenstein, Itamar, Philips/Respironics, Resmed, Somnodent. S. Zimmermann und K. Lederer und geben an, dass kein Interessenkonflikt besteht. Für den Inhalt und die Abfassung des Beitrags sind ausschließlich die Autoren verantwortlich. Für den Beitrag wurde keine finanzielle Unterstützung geleistet.

Für diesen Beitrag wurden von den Autoren keine Studien an Tieren durchgeführt. Die Studie wurde durch die Ethikkommission der Charité-Universitätsmedizin Berlin genehmigt (EA 1/288/10). Für die aufgeführten Studien gelten die jeweils dort angegebenen ethischen Richtlinien.

Open Access. Dieser Artikel wird unter der Creative Commons Namensnennung 4.0 International Lizenz veröffentlicht, welche die Nutzung, Vervielfältigung, Bearbeitung, Verbreitung und Wiedergabe in jeglichem Medium und Format erlaubt, sofern Sie den/die ursprünglichen Autor(en) und die Quelle ordnungsgemäß nennen, einen Link zur Creative Commons Lizenz beifügen und angeben, ob Änderungen vorgenommen wurden.

Die in diesem Artikel enthaltenen Bilder und sonstiges Drittmaterial unterliegen ebenfalls der genannten Creative Commons Lizenz, sofern sich aus der Abbildungslegende nichts anderes ergibt. Sofern das betreffende Material nicht unter der genannten Creative Commons Lizenz steht und die betreffende Handlung nicht nach gesetzlichen Vorschriften erlaubt ist, ist für die oben aufgeführten Weiterverwendungen des Materials die Einwilligung des jeweiligen Rechteinhabers einzuholen.

Weitere Details zur Lizenz entnehmen Sie bitte der Lizenzinformation auf http://creativecommons.org/ licenses/by/4.0/deed.de.

\section{Literatur}

1. Feierabend S, Rathgeb T, Reutter T (2018) Jugend, Information, Medien - Ergebnisse der JIM-Studie 2018. Media Perspekt 2018:14

2. Feierabend S, Rathgeb T, ReutterT (2019) Kindheit, Internet und Medien - Ergebnisse der KIM-Studie 2018. Media Perspekt 2019:10

3. Muñoz-Miralles R, Ortega-González R, LópezMorón MR et al (2016) The problematic use of Information and Communication Technologies (ICT) in adolescents by the cross sectional JOITIC study. BMC Pediatr 16:140
4. Dworak M, Schierl T, Bruns T et al (2007) Impact of singular excessive computer game and television exposure on sleep patterns and memory performance of school-aged children. Pediatrics 120:978-985

5. Kevitiyagala D, Finlay F, Baverstock A (2011) Question 1. What is the impact of computer games on sleep in children? Arch Dis Child 96:894-895

6. Van Den Bulck J (2004) Television viewing, computer game playing, and Internet use and self-reported time to bed and time out of bed in secondary-school children. Sleep 27:101-104

7. Gaina A, Sekine M, HamanishiSetal (2007) Daytime sleepiness and associated factors in Japanese school children. JPediatr 151:518-522.e1-4

8. Higuchi S, Motohashi Y, Liu Y et al (2005) Effects of playing a computer game using a bright display on presleep physiological variables, sleep latency, slow wave sleep and REM sleep. J Sleep Res 14:267-273

9. Weaver E, Gradisar M, Dohnt H et al (2010) The effect of presleep video-game playing on adolescent sleep. J Clin Sleep Med 6:184-189

10. Backhaus J, Junghanns K, Broocks $A$ et al (2002) Test-retest reliability and validity of the Pittsburgh Sleep Quality Index in primary insomnia. JPsychosom Res 53:737-740

11. Morin CM, Belleville G, Bélanger L et al (2011) The Insomnia Severity Index: psychometric indicators to detect insomnia cases and evaluate treatment response. Sleep 34:601-608

12. Johns MW (1991) A new method for measuring daytime sleepiness: the Epworth sleepiness scale. Sleep 14:540-545

13. Griefahn B, Künemund C, Bröde $P$ et al (2001) Zur Validität der deutschen Übersetzung des Morningness-Eveningness-Questionnaires von Horne und Östberg. Somnologie 5:10

14. Dalbert C (1992) Subjektives Wohlbefinden junger Erwachsener: Theoretische und empirische Analysen der Struktur und Stabilität. ZDiffer Diagn Psychol 13:207-220

15. Akerstedt T, Gillberg M (1990) Subjective and objective sleepiness in the active individual. Int J Neurosci 52:29-37

16. Iber C, Ancoli-Israel S, Chesson A et al (2007) The AASM manual for the scoring of sleep and associated events: rules, terminology and technical specifications. American Academy of Sleep Medicine, Westchester

17. Olds T, Ridley K, Dollman J (2006) Screenieboppers and extreme screenies: the place of screen time in the time budgets of 10-13 year-old Australian children. Aust NZJ Public Health 30:137-142

18. Lampert T, Sygusch R, Schlack R (2007) Nutzung elektronischer Medien im Jugendalter. Ergebnisse des Kinder- und Jugendgesundheitssurveys (KiGGS). Bundesgesundheitsblatt Gesundheitsforschung Gesundheitsschutz 50:643-652

19. Wolfson AR, Carskadon MA (1998) Sleep schedules and daytime functioning in adolescents. Child Dev 69:875-887

20. Shochat T, Flint-Bretler O, Tzischinsky O (2010) Sleep patterns, electronic media exposure and daytime sleep-related behaviours among Israeli adolescents. Acta Paediatr 99:1396-1400

21. Carskadon MA, Wolfson AR, Acebo C et al (1998) Adolescent sleep patterns, circadian timing, and sleepiness at a transition to early school days. Sleep 21:871-881

22. Taylor DJ, Jenni OG, Acebo C et al (2005) Sleep tendency during extended wakefulness: insights into adolescent sleep regulation and behavior. JSleep Res 14:239-244 


\section{Originalien}

23. Millman RP (2005) Excessive sleepiness in adolescents and young adults: causes, consequences, and treatment strategies. Pediatrics 115:1774-1786

24. Roenneberg T, Kuehnle T, Pramstaller PP et al (2004) A marker for the end of adolescence. Curr Biol 14:R1038-R1039

25. Tarokh L, Saletin JM, Carskadon MA (2016) Sleep in adolescence: physiology, cognition and mental health. Neurosci Biobehav Rev 70:182-188

26. Ohayon MM, Carskadon MA, Guilleminault C et al (2004) Meta-analysis of quantitative sleep parameters from childhood to old age in healthy individuals: developing normative sleep values across the human lifespan. Sleep 27:1255-1273

27. Li S, Jin X, Wu S et al (2007) The impact of media use on sleep patterns and sleep disorders among school-aged children in China. Sleep 30:361-367

28. Dijk DJ, Cajochen C, Borbély AA (1991) Effect of a single 3-hour exposure to bright light on core body temperature and sleep in humans. Neurosci Lett 121:59-62

29. Komada Y, Tanaka H, Yamamoto Y et al (2000) Effects of bright light pre-exposure on sleep onset process. Psychiatry Clin Neurosci 54:365-366

30. Bhattacharyyia P, Das S et al (2017) Exposure to video games shortens simple visual reaction time-a study in Indian school children. Ann Appl BioSci 4:5

Hier steht eine Anzeige.

\section{Springer}

